\title{
Additive manufacturing methods and materials for electrokinetic systems
}

Katarina Bengtsson 
During the course of the research underlying this thesis, Katarina Bengtsson was enrolled in Forum Scientum, a multidisciplinary doctoral program at Linköping University, Sweden

Additive manufacturing methods and materials for electrokinetic systems

(C) Katarina Bengtsson

Linköping Studies in Science and Technology. Thesis No. 1724

ISBN: 978-91-7685-988-9

ISSN 0280-7971

Printed by LiU-Tryck, Linköping, Sweden, 2015 


\section{Abstract}

Fabrication of miniaturized devices is usually time-consuming, costly, and the materials commonly used limit the structures that are possible to create. The techniques most often used to make microsystems involve multiple steps, where each step takes considerable time, and if only a few systems are to be made, the price per device becomes excessive. This thesis describes how a simple syringebased 3D-printer, in combination with an appropriate choice of materials, can reduce the delay between design and prototype and simplify fabrication of microsystems. This thesis suggest two types of materials that we propose be used in combination with 3D-printing to further develop microsystems for biology and biochemistry.

Analytical applications in biology and biochemistry often contain electrodes, such as in gel electrophoresis. Faradaic (electrochemical) reactions have to occur at the metal electrodes to allow electron-to-ion transduction through an electrolyte-based system to drive a current when a potential is applied to the electrodes in an electrolyte-based system. These electrochemical reactions at the electrodes, such as water electrolysis, are usually problematic when miniaturizing devices and analytical systems. An alternative to metal electrodes can be electrochemicallyactive conducting polymers, e.g. poly(3,4-ethylenedioxythiophene) (PEDOT), which can be used to reduce electrolysis when driving a current through water-based systems. Paper 1 describes gel electrophoresis where the platinum electrodes were replaced with the conductive polymer PEDOT, without affecting the separation.

Manufacturing and prototyping of microsystems can be simplified by using 3Dprinting in combination with a sacrificial material. A sacrificial template material can further simplify bottom-up manufacturing of more complicated forms such as protruding and overhanging structures. We showed in paper 2 that polyethylene glycol (PEG), in combination with a carbonate-based plasticizer, functions well as a 3D-printable sacrificial template material. PEG2000 with between $20 \mathrm{wt} \%$ and $30 \mathrm{wt} \%$ ethylene carbonate or propylene carbonate has properties advantageous for 3D-printing, such as shear-thinning rheology, mechanical and chemical stability, and easy dissolution in water. 


\section{Preface}

This licenciat thesis, which highlights and summarizes my work between November 2011 and January 2015, will be a part of my Ph.D. thesis. The work has been performed in the Transport and Separations Group, within the Division of Surface Physics and Chemistry at the Department of Physics, Chemistry and Biology at Linköping University

Financial support was provided by the Swedish Research Council (Vetenskapsrådet) grants 2007-3983, 2008-7537, and 2011-6404 and by Linköping University through a "Career Contract". 


\section{Acknowledgments}

Här är det plats för ett och annat tack till alla er runt omkring:

Min handledare, Nate Robinson

"Gruppen": Sara, Per, Abdel, Marek

> Surfphys med min biträdande handledare Mats Fahlman i spetsen och Kerstin Vestin som håller koll.

Daniel Brandell och Jonas Mindemark

$>$ Forum Scientiums alla medlemmar

> Kaffeklubbens alla gamla och nya medlemmar

$>$ Firandeklubben som i år nog får sig ett uppsving.

$>$ Familj och vänner utanför akademin som gör sitt bästa för att försöka förstå. 


\section{Included papers}

1. Conducting polymer electrodes for gel electrophoresis

Katarina Bengtsson, Sara Nilsson, Nathaniel D. Robinson

PLOS ONE, 2014

doi:10.1371/journal.pone.0089416

Author contribution: Tested and evaluated large PEDOT electrodes which were then incorporated into gel electrophoretic systems. Demonstrated separation equivalence.

2. Sacrificial template materials for syringe-based 3D-printing Katarina Bengtsson, Jonas Mindemark, Daniel Brandell, Nathaniel D. Robinson

Submitted

Author contribution: Developed sacrificial material, analyzed data from rheology and DSC experiments. Tested printing properties and performed dissolution experiments. Structured manuscript. 


\section{Contents}

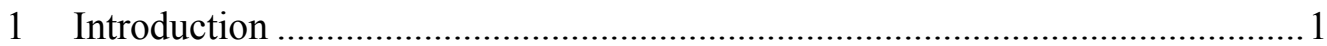

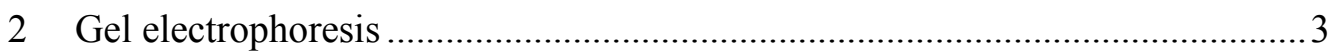

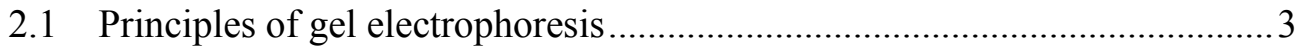

2.2 Impact of electrochemical reactions when miniaturizing electrokinetic

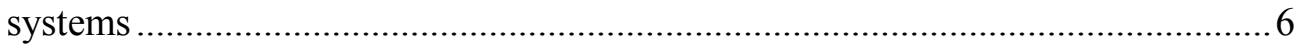

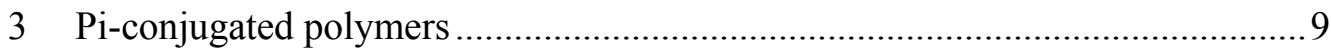

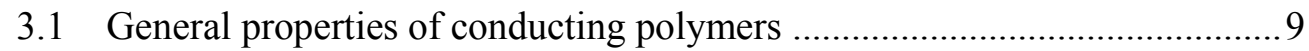

3.2 Electrochemical oxidation and reduction of pi-conjugated polymers ......... 10

3.3 Limitations of conductive polymers …………........................................ 12

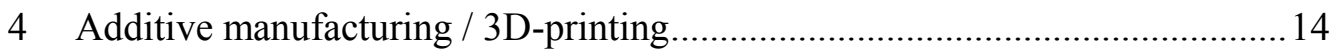

4.1 Basic principles of 3D-printing ………................................................... 14

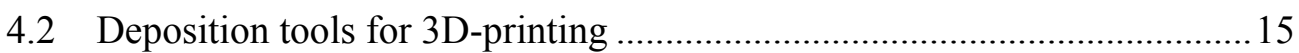

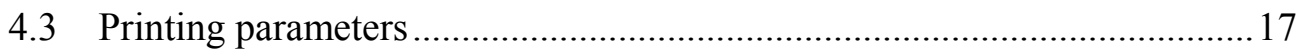

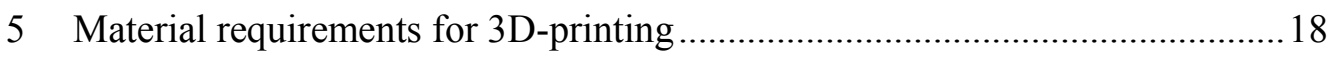

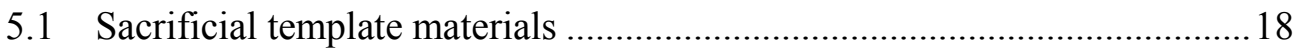

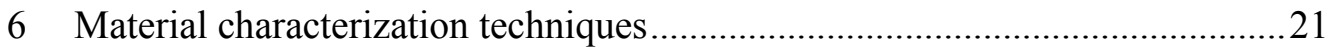

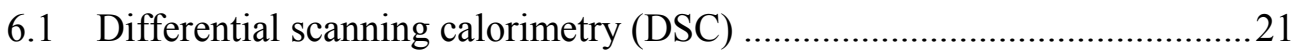

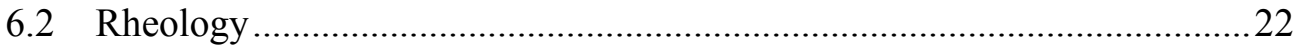

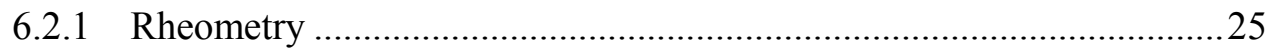

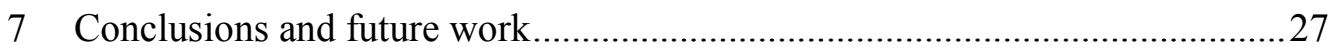

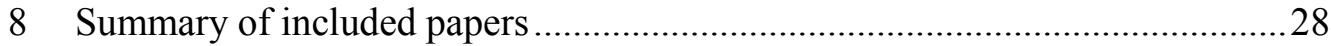

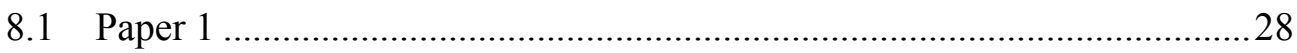

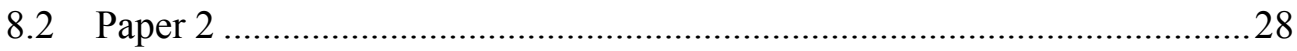





\section{Introduction}

A Lab On a Chip (LoC) is a credit card-sized or smaller device where many of the functionalities that can be found in a chemistry lab are synthesized. Examples of simple and user-friendly devices that exist today are the glucose sensor and the home-pregnancy test, which both have microstructures that guide the sample to a certain spot for analysis. LoCs generally handles fluid volumes of nanolitres $\left(10^{-9}\right)$ and below, which means that only a small drop (estimated to $\sim 40000 \mathrm{~nL}$ ) of blood is enough for multiple analysis in a single system. ${ }^{1}$ There are several reasons for miniaturizing an analytical system, e.g. increased rate of analysis, highly parallelized sample/analysis, portability, no need for multiple setups, and small sample sizes, leading to cost-efficient analyses compared to larger systems. These properties make LoCs systems useful in areas with limited resources and by making self-supporting analytical systems can tests be available for more people. There are several methods and materials available to produce LoCs, e.g. photolithography, soft lithography, and injection molding, which are all useful to mass-produce microstructures. But, for small volumes of devices or prototypes, these techniques are both time-consuming and costly.

Materials are required when building anything, be it a house, a car, a pacemaker, analytical system for proteins, or a cell culturing chamber. The desired properties of the final construction help determine which materials should be used. For applications in biology, the materials must be biocompatible, and in some cases transparent, e.g., devices monitored in microscopes. For analytical applications in, e.g. biochemistry, it is necessary to integrate electrodes to the microsystem. Metal electrodes are rigid, expensive and Faradaic (electrochemical) reactions at the electrodes usually generate gas and $\mathrm{pH}$ changing ion when used to apply a potential to a water-based system, which can be problematic in microsystems. An alternative to metal is conducting polymers, which are flexible, cheap, easily manufactured, and electrochemically active (see chapter 3 about conductive polymers). An example where conducting polymer electrodes can be used is gel electrophoresis. Paper 1 shows how the conducting polymer PEDOT can replace platinum electrodes without negatively influencing protein separation in gel electrophoresis. 
As mentioned previously, techniques for making LoCs, e.g. photolithography and injection molding, are both time-consuming and costly when making a small number of devices. These techniques are also limited to certain types of material and structures. 3D-printing on the other hand makes single objects which easily can be adapted from time to time with a combination of various materials. ${ }^{2}$ Interest in the field of 3D-printing/additive manufacturing is increasing, and many believe that it will revolutionize how objects are manufactured. There are already many different applications and products available made by 3D-printing. Cheap and open-source 3D-printers have increased the number of users where interested, creative, and innovative people can fabricate individualized products by themselves. In this work, 3D-printing is seen as a potential tool for bottom-up manufacturing of LoC devices, achieving structures difficult or impossible to produce with more traditional manufacturing techniques. Sacrificial template materials can further advance 3D-printing and increase the complexity of the structures that can be built. Paper 2 describes a sacrificial template material for syringe-based 3D-printing to increase and simplify production of a wider range of structures. This thesis focuses on methods, and materials, which are intended to be used in assays within different areas of biology and biochemistry, where the choice of method and material can facilitate and solve some of the challenges associated with miniaturization and prototype manufacture. 


\section{Gel electrophoresis}

The following chapter describes the basic principles of gel electrophoresis and challenges encountered when miniaturizing gel electrophoresis systems, particularly those associated with the electrochemical reactions taking place at the electrodes.

Gel electrophoresis is a method to separate charged macromolecules, such as proteins, DNA, RNA, and amino acids, in a gel across which an electric field is applied. ${ }^{3-5}$ Gel electrophoresis can be used as a purification step, e.g., after PCR, in order to improve the efficiency of subsequent analysis. This technique is a standard analytical method in biochemistry, molecular biology and forensic science.

\subsection{Principles of gel electrophoresis}

Gel electrophoresis separates charged molecules based on differences in their mobility in an electric field. ${ }^{4,5}$ The mobility of each molecule depends on its charge distribution, structure and size. The separation is influenced by the type of gel used and the applied electric field. A schematic setup can be seen in Figure 1. A potential is applied between two electrodes, typically made of platinum, that are placed in buffer at opposite sides of the gel, e.g. an agarose gel or polyacrylamide gel. 


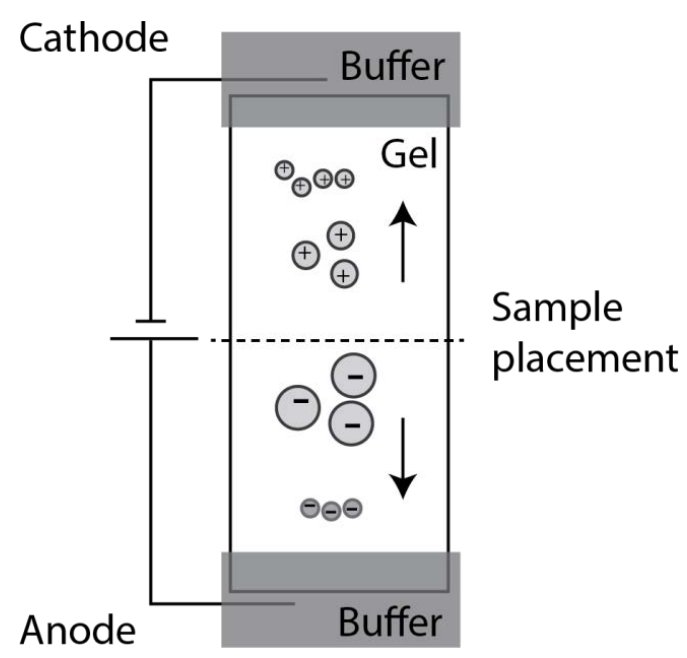

Figure 1 Schematic setup of gel electrophoresis, where differently charged species has been separated depending on their size. The sample, a mixture of the charged species, is placed in the middle of the gel (dashed line) from which the charged species will start to migrate through the gel. Cations (positively charged ions) move towards the cathode (negatively charged electrode) and anions (negatively charged ions) move towards the anode (positively charged electrode) with different migration rates.

The applied potential creates an electric field in which all anions (negatively charged species) move towards the anode, which is positively charged, and all cations move towards the cathode. The applied potential starts Faradaic (electrochemical) reactions (an oxidation reaction at the anode and a reduction reaction at the cathode) that drive an ionic current through the gel. Without Faradaic (electrochemical) reactions at the electrodes, the ions would accumulate at the interface between the gel and the electrode and shield the bulk of the gel from the electric field - more about this later. The gel is partially depleted from ions since they move in the gel towards the oppositely charged electrode. The electrical resistance in the gel is thereby increased leading to a decreased current when a constant potential is applied. The gel has a sieving effect, where larger molecules move more slowly and smaller molecules move more swiftly. The migration rate of species through the gel is related to the current through the gel. The migration rate can be kept constant by running the process at a constant current, where the potential increases with time during the separation. Gel electrophoresis is thereby either run at a constant applied potential or at a constant current. The separation in 
paper 1 was performed using a constant current where the applied potential ranged from $60 \mathrm{~V}$ up to $250 \mathrm{~V}$ over a $4 \mathrm{~cm}$ long gel.

The most common gels used in electrophoresis are agarose and polyacrylamide. The different gels are optimized for different types of samples and procedures. Agarose is usually appropriate for large proteins, and DNA, while polyacrylamide gels are used for smaller proteins, fragments of DNA, and peptides. ${ }^{5}$ The choice of gel also influences what can be done with the sample after the separation (blotting, etc.). For example, agarose gels allow for easy retrieval of the sample by simply melting the gel. When making agarose gel, there is little control over the gel's porosity, while the porosity/density of a polyacrylamide gel can be reliably and repeatedly determined. ${ }^{3,5}$ A polyacrylamide gel is formed by mixing acrylamide and bisacrylamide together using a radical to initiate polymerization. Varying the concentration of acrylamide and bisacrylamide, influences the resulting gel density: the higher the concentration of acrylamide, the higher the density of the gel and the more suitable it is for separation of smaller sample constituents. The same goes for agarose gel, where higher agarose concentration leads to a denser gel, but dense agarose is not as dense as polyacrylamide. The choice of gel material and the porosity of the gel also affect how well the bands/proteins can be resolved/separated in the gel. Optimizing the gel in this manner is outside the scope of this thesis. A commercially available polyacrylamide gel with a density gradient was used in paper 2 .

There are different methods of employing gel electrophoresis. Isoelectric focusing (IEF) uses a gel with varying $\mathrm{pH}$, and separates native proteins with respect to their isoelectric point, the $\mathrm{pH}$ at which the protein's net-charge is zero. When the potential is applied, the electric field starts electromigration of the ions and creates a $\mathrm{pH}$-gradient in the gel. The sample components migrate in the gel until they each reach an environment where the $\mathrm{pH}$ matches the component's isoelectric point. This type of gel electrophoresis maintains the native structure of the protein for further analysis or use. Another method is sodium dodecyl sulfate polyacrylamide gel electrophoresis (SDS-PAGE), which separates denatured proteins depending on their mass. Dodecyl sulfate denatures proteins, adding a negative net-charge. SDSPAGE separations are therefore always run from cathode to anode. The amount of SDS that interacts with a protein is proportional to the number of amino acids. ${ }^{4}$ The ratio is approximately one molecule of SDS per two amino acids. The mobility of 
the denatured protein will, for the most part, be linearly logarithmic to the mass of the protein. This can be compensated by using gels with a density gradient which helps improve the separation of proteins within a shorter distance.

\subsection{Impact of electrochemical reactions when miniaturizing electrokinetic systems}

There are several challenges to miniaturizing disposable gel electrophoresis systems, here described in respect to the electrodes, which paper 1 suggests a solution to. Electrodes are usually made out of metal in gel electrophoretic systems, e.g. Pt or $\mathrm{Au}$, which are reused between runs, due to their price. The reuse introduces the risk of cross-contamination between successive runs.

The electric field in the gel is maintained by electrochemical reactions at the electrodes (see Figure 2). The main electrochemical reaction at the electrodes in a water-based system is water electrolysis, which produces gas (oxygen or hydrogen gas) and $\mathrm{pH}$-changing species, like hydrogen and hydroxyl ions. Water is either oxidized into $\mathrm{O}_{2}(\mathrm{~g})$ and hydrogen ions or reduced into $\mathrm{H}_{2}(\mathrm{~g})$ and hydroxyl ions (see half-reactions 1 and 2).The oxidation takes place at the anode while the reduction takes place at the cathode.

$$
\begin{aligned}
& 2 \mathrm{H}_{2} \mathrm{O} \rightarrow \mathrm{O}_{2(\mathrm{~g})}+4 \mathrm{H}^{+}+4 \mathrm{e}^{-} \\
& 2 \mathrm{H}_{2} \mathrm{O}+2 \mathrm{e}^{-} \rightarrow \mathrm{H}_{2(\mathrm{~g})}+2 \mathrm{OH}^{-}
\end{aligned}
$$




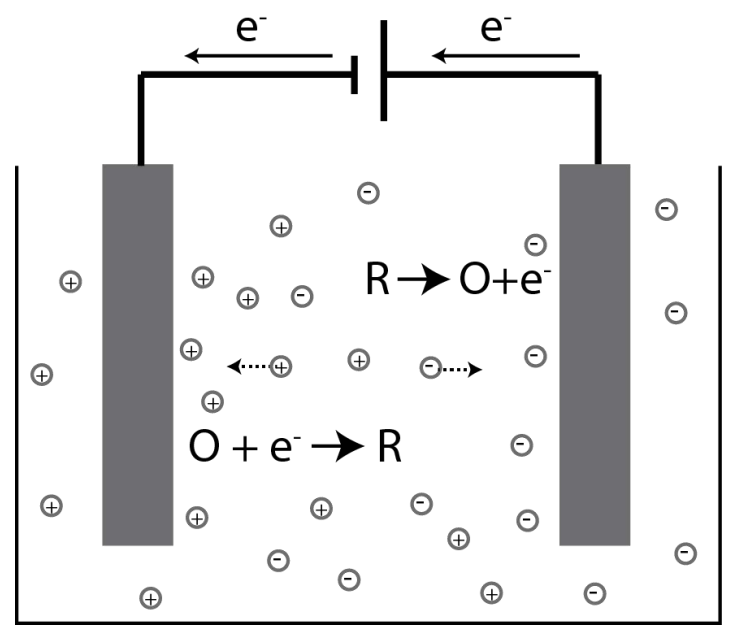

Figure 2 General electrochemical cell with metal electrodes immersed in electrolyte. Reduction occurs at the cathode (right-hand side) and oxidation at the anode (left), which generates a current through the electrochemical cell. Any ions in the electrolyte move towards the oppositely-charged electrode.

The amount of water that is consumed by electrolysis is proportional to the current through the gel during the separation. The higher the current, the more electrolysis has to occur, which leads to faster consumption of water and generation of $\mathrm{pH}$ changing species (see reactions 1 and 2). The consumption of water by electrolysis risks drying the gel, which then increases the resistance, thereby decreasing the separation rate and preventing migration through the gel. These effects can be reduced by increasing the amount of buffer available. However, the amount of buffer is limited in miniaturized systems to the scale of microliters or less, which renders them more sensitive to drying and $\mathrm{pH}$ changes than larger systems.

However, microsystems maintain a higher electric field with lower current, due to their small dimensions leading to higher resistances compared to large systems. By decreasing the current, less water electrolysis is required to maintain the electric field, producing fewer protons, hydroxyl ions and gas. The gas that forms is easily dissipated in large, open systems, but can get trapped in microsystems, thereby breaking the connection through the electrolyte or the gel. The interface between electrode and electrolyte is also affected by any gas that remains there, reducing the active electrode area available to drive the electrochemical reactions.

Miniaturization is facilitated by replacing water electrolysis with another Faradaic 
reaction. This reduces the previously mentioned undesired products, like protons and hydroxyl ions, further described in chapter 3.2.

A constant $\mathrm{pH}$ is important since a protein's charge depends on the $\mathrm{pH}$. $\mathrm{pH}$ changes can alter the migration speed or even direction of the protein through the gel, for example used in the IEF setup previously mentioned, but causes problems in other setups, e.g. reduces resolution between the constituents. For example, all proteins in SDS-PAGE have a negative charge, independent of the native proteins' charge, which is assumed to be unchanged at different $\mathrm{pH}$ and thereby less sensitive to $\mathrm{pH}$ changes. It is therefore the water consumption and gas formation from water electrolysis that limit miniaturization of SDS-PAGE. 


\section{Pi-conjugated polymers}

Polymers are molecules in which a subunit, also called monomer, is repeated. The monomer is the smallest group that can be repeated to form the entire polymer. Most polymers are good insulators, and are used in various applications to shield conductive materials from short-circuiting. This was the case until 1977 when it was discovered that the conductive properties of the semi-conducting pi-conjugated polymer trans-polyacetylene improved when using halogens during production ${ }^{6}$, which can be considered to be the start of the research field of conducting polymers. Heeger, MacDiarmid, and Shirakwa were awarded the Nobel prize in chemistry in 2000 for their work on conducting polymers.

\subsection{General properties of conducting polymers}

A pi-conjugated polymer has a carbon backbone with alternating double- and single-bonds, see Figure 3. The neighboring pi-bonds (one of the bindings in a double-bond) partially overlap. The overlap allows electrons to move between different pi-bonds along the pi-conjugated backbone of the polymer. This conducting pathway conducts current within the polymer similar to the way a metal does. Examples of conductive polymers are polypyrrol, polyacetylene, and polythiophenes, specifically poly(3,4-ethylenedioxythiophene) (PEDOT) (see Figure 3). Throughout this thesis, the terms conductive polymer and conducting polymer are used interchangeably.

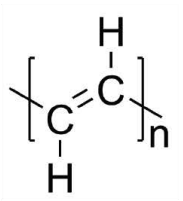

polyacetylene

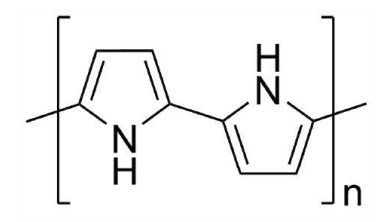

polypyrrol

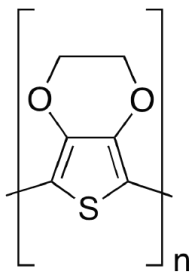

poly(3,4-ethylenedioxythiophene) (PEDOT)

Figure 3 Examples of conductive polymers and their chemical structures 
Conductive polymers have several advantages over metals and inorganic semiconductor materials: they are flexible, cheap, easily manufactured and modifiable, and many are biocompatible ${ }^{7-9}$. These properties makes conductive polymers useful in various applications, e.g. solar cells, electrodes in microsystems, antistatic coatings, and actuators. Biological applications benefit from flexible materials that are less rigid than e.g. metals and have a consistency similar to tissue and cells. Methods for depositing conductive polymers are, e.g. solution processes and electropolymerization. Solution process deposition includes several techniques such as spray-painting, drop-casting, spin-coating, and printing such as inkjet, screen- and 3D-printing. ${ }^{10}$

\subsection{Electrochemical oxidation and reduction of pi-conjugated}

\section{polymers}

Many conductive polymers are electrochemically active, meaning that they can be oxidized or reduced when a potential is applied and ions are present. The oxidation for a general pi-conjugated polymer follows reaction scheme 3 , where $\mathrm{P}^{0}$ is the neutral and undoped form of the conjugated polymer and $\mathrm{X}^{-}$is a counter ion. Applying an anodic potential oxidizes the polymer into $\mathrm{P}^{+}$, which results in the formation of a complex with the counter ion, stabilizing the oxidized form of the polymer. Conducting polymers with such a positive charge are said to be $p$-doped. There are also polymers that can be reduced to have a negative net charge, $n$-doped, with a positive counter ion instead, although these are less common. Reduction follows the reverse path of the half-reaction 3 .

$$
\mathrm{P}^{0}+\mathrm{X}^{-} \rightarrow \mathrm{P}^{+} \mathrm{X}^{-}+\mathrm{e}^{-}
$$

Pi-conjugated polymers are useful as electrodes in water-based electrolyte systems, such as gel electrophoresis. Water electrolysis (described in chapter 2.2) is usually the dominant reaction when applying a potential to two metal electrodes immersed in water. The current through the electrolyte is maintained by the electrochemical reactions at the electrodes, where the metal functions as an electron-transfer material to the oxidation or reduction half-reaction (see Figure 2). There is a potential difference between the half-reactions of oxidation and reduction of water which defines the electrochemical stability window of the electrolyte. The potential difference between two electrodes of the same conducting polymer is zero. The redox reaction of pi-conjugated polymers at the electrodes is reversible between the 
polymers's oxidized (e.g. positively charged state) and reduced states (e.g. neutral state). The electrochemical oxidation/reduction of the polymers starts when a small potential is applied between the electrodes, which drives a current through the electrochemical cell. Ideally would no water electrolysis occur and the only reaction would be oxidation/reduction of the polymer electrodes to drive the current through the water-based electrochemical cell. This can be seen for a water-based electrochemical cell, where the resulting current is larger when using polymer electrodes compared to metal electrodes when applying a potential within the electrochemical stability window of water. The dominating electrochemical reaction at an electrode can therefore be altered to the oxidation/reduction of conjugated polymers instead of water electrolysis by changing the electrode material.

The conjugated polymer poly(3,4- ethylenedioxythiophene) (PEDOT) has been used in several applications and was also used in paper 1 in combination with the counter ion polystyrene sulfonate (PSS). PEDOT is stable in its oxidized state, $\left(\mathrm{PEDOT}^{+}\right)$, and is highly electrically-conductive compared to other polymers, easy to process and manufacture, biocompatible $\mathrm{e}^{7,8}$, and electrochromatic ${ }^{11}$. Oxidation (reaction at anode) of PEDOT is seen in half-reaction 4, where PEDOT ${ }^{0}$ is the neutral and reduced form of the conjugated polymer. $\mathrm{M}^{+}$is any cation which can move between the polymer film and the electrolyte. PEDOT is often manufactured and sold with the counter ion polystyrene sulfonate ( $\mathrm{PSS}^{-}$), which stabilizes the PEDOT in aqueous medium leading to a dispersion denoted PEDOT:PSS, since PEDOT otherwise is not water-soluble. ${ }^{12}$ Oxidation and reduction of PEDOT was used to replace water electrolysis to generate the electric field over the gel during SDS-PAGE in paper 1 without affecting the performance of the separation method. PEDOT electrodes will initially consist of both doped and undoped polymer chains making the electrode functions as either cathode or anode. By replacing the metal electrodes with PEDOT:PSS, we expect (but did not directly show) the effects of water electrolysis to be reduced. It has previously been shown that PEDOT:PSS can be used to mitigate water electrolysis. ${ }^{13}$

$$
\text { PEDOT }^{0}+\mathrm{M}^{+} \mathrm{PSS}^{-} \rightarrow \text { PEDOT }^{+} \mathrm{PSS}^{-}+\mathrm{M}^{+}+\mathrm{e}^{-} \quad(4)
$$

A conjugated polymer film functions as an electron-to-ion transducer, since the transfer of electrons leads to ion movement between the polymer film and the 
electrolyte during oxidation and reduction (see Figure 4). The movement of ions in conjugated polymers can therefore be used as a delivery system of biomolecules or ions to e.g. cells in biology applications. ${ }^{8,14}$ The movement of ions also causes the polymer to swell, since the ions draw water into the film and actuators based on this principle have been demonstrated..$^{15,16}$

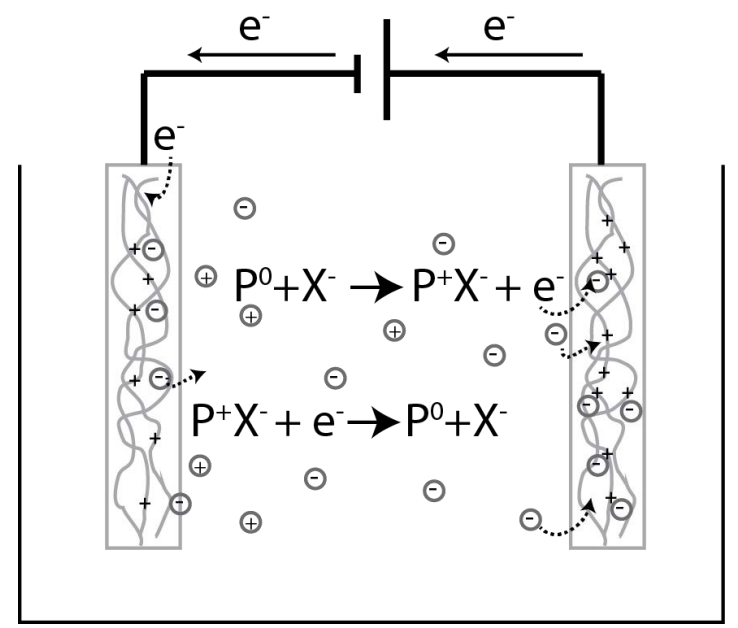

Figure 4 Electrochemical cell with polymer electrodes immersed in electrolyte. Oxidation and reduction at the electrodes generates a current through the electrochemical cell. The polymer oxidizes at the anode and anions move in to the polymer film from the electrolyte. The polymer reduces at the cathode and ions move into the electrolyte from the polymer film.

\subsection{Limitations of conductive polymers}

The limited charge capacity of conductive polymers follows from the finite number of polymer molecules available for oxidation and reduction, which initially consist of both charged and uncharged polymer chains. When these are consumed, the polymer cannot undergo further oxidation or reduction, meaning that it behaves like an electrochemically-inactive metal electrode, driving a current through the circuit via another Faradaic reaction (electrolysis). This will then start the production of gas (hydrogen and oxygen) and $\mathrm{pH}$ changing species (hydrogen ions and hydroxyl ions). It is therefore important to properly design the size of the electrodes for a given system to avoid depleting the electrochemically active part of the polymer. 
Many conducting polymers are also susceptible to over-oxidation, rendering the polymer non-conductive. Over-oxidation describes an electrochemical process that oxidizes a polymer beyond the p-doped state. Once over-oxidized, the conjugated backbone is damaged, and its conducting properties vanish. Generally, this occurs when an excessive potential is applied to the conductive polymer. Keeping the applied potential below a limit specific to each polymer reduces the likelihood of over-oxidation. However, higher potentials can be applied if the design of the system results in an applied potential between the electrode and electrolyte that is less than the over-oxidation potential. Potentials up to $250 \mathrm{~V}$ were applied in paper 1, using PEDOT:PSS electrodes without over-oxidizing the polymer. Here the largest potential drop occurred over the gel, while the potential drop at the interface between the electrodes and gel were smaller than the over-oxidation potential. 


\section{Additive manufacturing / 3D-printing}

3D-printing, as an additive manufacturing method, has rapidly become a useful technique for making small quantities of individual systems, customized prototypes or prostheses. 3D-printers use a bottom-up approach, where material is added layerby-layer to an object. Over time, 3D-printers have evolved from expensive tools available only to a few experts to low-cost machines accessible by everyone. ${ }^{2,17}$ There are several types of 3D-printers with different deposition methods, described in the following chapter.

3D-printers have decreased the time required to convert a virtual 3D-model into an actual object, allowing testing of different designs and prototypes during the same time it previously took to make a first prototype. ${ }^{2,17}$ The quality of the final printed object depends on the material, calibration of the deposition tool's parameters, and the type of printer used. Lower quality objects are made quickly; however, with increased demands on quality and refined structures, comes an increase in production time and higher demands on calibration. Open-source and cheap printers usually produce lower-quality objects, which can be sufficient for prototyping and research. These types of printers allow a lot of freedom in terms of the use of various (and non-commercial) materials and the possibility to modify the printer to suit specific applications. The dimensions of the printed object can be in the range of a few millimeters up to $25 \mathrm{~cm}$ for a cheap desktop printer.

\subsection{Basic principles of 3D-printing}

The basic principle of additive manufacturing is that an object is built by adding material (layer-by-layer) to replicate a digital 3D-model. Figure 5 illustrates a 3Dprinter where the deposition tool is a syringe filled with building material. The deposition tool translates in the $\mathrm{x}$ - and $\mathrm{y}$-directions (horizontal plane) while depositing material by translating the piston in the syringe. To create multi-layered structures, either the table or the deposition tool is moved in the z-direction, creating space for the next layer. 


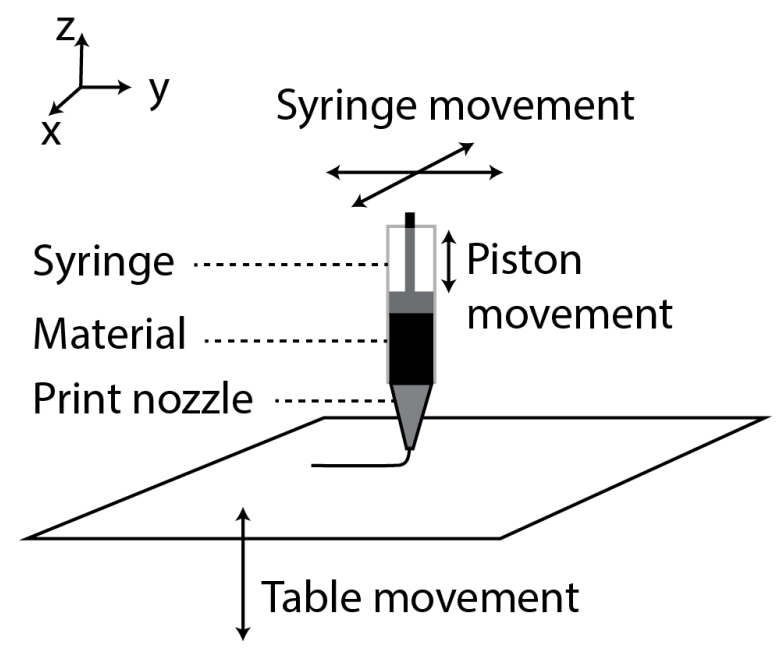

Figure 5 Basic principle of 3D-printing, illustrated with a syringe filled with the construction material as the deposition tool. Movement of a piston extrudes material while the syringe moves in the $x$ - and $y$-direction to form the shape of the object. For each layer, the table moves down (in the z-direction).

\subsection{Deposition tools for 3D-printing}

The choice of deposition tool varies depending on the material and technique used to build an object. There are 3D-printers that melt materials by sintering, polymerizing materials with laser- or UV-light, or adding material by extrusion. Sintering-based printers form an object by printing a glue, solvent or melting a powder into the desired 3D-structure. The structure is formed embedded within the powder, which supports the object while it is being printed. Sintering-based printers provide more freedom when designing overhanging and protruding structures compared to other 3D-printers, since the object is surrounded by the powder which supports printed structures. Materials for sintering-based printers have to be compatible with the glue or melting process, which makes many biomaterials unsuitable. UV/laser-based printers require a material that is photo-curable into a solid. The structure is built by directing light in the shape of the structure into a basin of photo-curable material. Extrusion-based 3D-printers add material bottomup to an object, and can have various extrusion tools that are often specific to a certain type of material. Thermoplastic printers extrude plastic filament heated to $60{ }^{\circ} \mathrm{C}$ or more. The increased temperature precludes the use of materials that are 
easily destroyed at these temperatures, including many biological materials. This is the type of printer most familiar to the general public, mainly because it represents the largest group of cheap and open-source printers available, e.g. RepRap ${ }^{18,19}$, which evolved into the commercial printer MakerBot. The plastic filaments used consist of, e.g., poly lactic acid (PLA), acrylonitrile butadiene styrene (ABS), and polyvinyl alcohol (PVA). Another type of additive deposition tool is the inkjet printer, which uses a deposition head similar to those commonly found in commercial desktop document printers. This type of deposition tool requires "ink" with low viscosity. In comparison, syringe-based printers can extrude both high and low viscosity material. ${ }^{20}$ Syringe-based printers extrude material through a tip attached to a syringe barrel, where the pressure on the piston can be controlled. Examples of materials printed with this type of printer are silicones, biomaterials including cells, frosting, and conductive polymers. ${ }^{2,20-23}$ An object composed of several types of materials can be constructed during a single printing run by simultaneously using multiple syringe barrels. The limits on the size of the object printed depend on the tip size, the material properties and to the accuracy of the printer. In paper 2, a material was developed for the syringe-based printer in Figure 6.

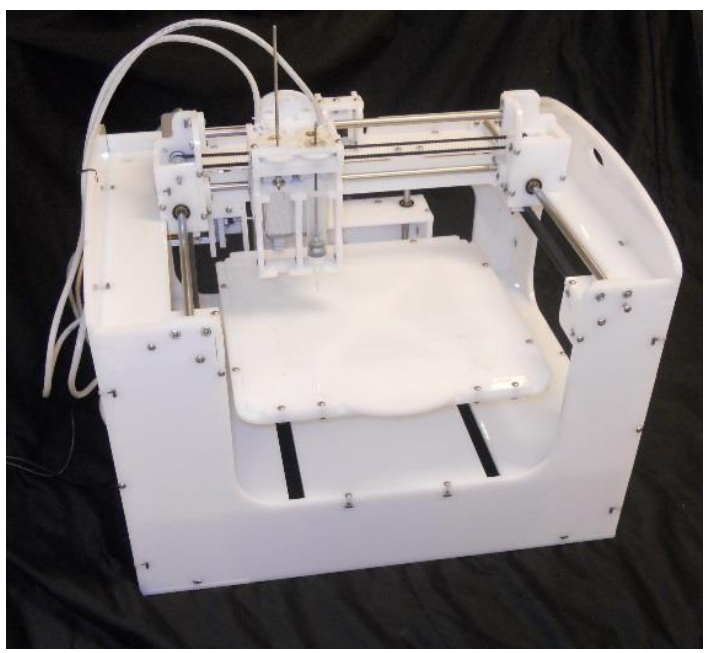

Figure 6 Syringe-based 3D-printer (Fab@home Model 3, Seraph Robotics Inc), photo by K. Bengtsson. 


\subsection{Printing parameters}

There are several parameters that govern extrusion during 3D-printing, e.g. the size of the nozzle, ambient temperature, material viscosity, nozzle translation speed, extrusion rate, which all influence the final product. Three settings adjusted during syringe-based 3D-printing are: deposition rate, initial pressure increase, and the final release of pressure on the material. These parameters have to be adjusted and optimized for each set of materials to get an even, straight structure with a crosssection similar to the nozzle without any gaps in the printed lines. The deposition rate is the rate of (continuous) flow of material during printing, and is usually specified relative to the translation rate of the nozzle. To initiate extrusion, the initial pressure created by the piston is increased (hereafter referred to as pushout), and the pressure is released (later referred to as suckback) to halt the flow. To initiate and stop flow of a viscous material, a larger pushout and suckback are required, compared to that required for a low viscosity material. The deposition rate and tip diameter influence the shear rate that a material is exposed to during printing and were used to estimate shear rates, as described in chapter 6.2. Chapter 5 includes a specification of properties that make a material suitable for syringebased 3D-printing. 


\title{
5 Material requirements for 3D-printing
}

The next step for further advancement of 3D-printing is the development of new materials with properties desirable for good printing quality. A material suitable for 3D-printing should be compatible with the deposition tool as well as the application. Material properties suitable for syringe-based printing for applications in biology and biochemistry are described in Table 1.

Table 1 Material properties desirable for syringe-based printing for applications in biology and
biochemistry.

\begin{tabular}{lll}
\hline Property & Definition & Need \\
\hline Biocompatibility & $\begin{array}{l}\text { A material that can coexist with living } \\
\text { tissue and cells, which has low or no } \\
\text { toxic effect in the biological application. }\end{array}$ & $\begin{array}{l}\text { Devices used in biology and biochemistry } \\
\text { typically need to perform their functions } \\
\text { without disturbing the tissues, cells, or } \\
\text { biomolecules under study. }\end{array}$ \\
\end{tabular}

Chemical stability The material has a consistent chemical structure and is inert when in contact with other materials.
Predictable behavior while printing and handling. Possibility to combine multiple materials in a device without undesired reactions.
Mechanical stability

Sufficient consistency for the mater
maintain its form when printed,
including the support of subsequent

layers and structures. Viscosity $>20 \mathrm{~Pa} \cdot \mathrm{s}$
3D-printing requires multiple layers to be patterned, where underlying structures support additional layers. High mechanical stability of the final printed object allows it to be handled without being deformed or otherwise damaged.
Thermal stability

The material should be stable and have consistent viscosity in the relevant temperature range during fabrication, use and handling.
Minimal changes of viscosity in the temperature range of $20 \pm 5^{\circ} \mathrm{C}$. Predictable behavior while printing and handling without temperature control. Many biological applications require temperature stability near $37^{\circ} \mathrm{C}$.

\subsection{Sacrificial template materials}

\begin{abstract}
A sacrificial material is a temporary support that allows for manufacture of more complicated printed structures in combination with other materials. It can, for example, facilitate the bottom-up construction of over-hanging, protruding and/or hollow structures, that otherwise are difficult to make with certain 3D-printers, e.g. syringe-based printing. A sacrificial material should be easy to remove, for
\end{abstract}


example by dissolution in water, but also maintain its printed structure during all steps until removal. For example, it must not dissolve or collapse when acting as a support during molding or during the addition of layers of another material.

Sacrificial template materials for biological applications are preferably water-soluble with biocompatible residues. Sugars ${ }^{24}$, poloxamer $^{25}$ and polyethylene glycol (PEG) ${ }^{22}$ are examples of materials that have been used as sacrificial template materials in biological applications.

The polymer polyethylene glycol, PEG, (also known as polyethylene oxide, PEO, see Figure 7) is available in a range of molecular weights. The consistency of the polymer ranges from liquid to crystalline (wax-like) with increasing chain length. The mechanical stability and printing properties can therefore be altered by choosing an appropriate molecular weight. The choice of molecular weight, i.e. the number $n$ in Figure 7, can also be optimized for dissolution of the printed PEG structure. Lower molecular weight PEG is more easily dissolved than higher molecular weight varieties. The dissolution of PEG can be improved by increasing the temperature, where shorter chains are affected more due to their lower melting temperature. PEG has been used in many different kinds of applications such as plasticizers ${ }^{26}$, hydrogels ${ }^{27}$, batteries ${ }^{28}$, cosmetics, laxatives and drug delivery systems. ${ }^{29,30} \mathrm{PEG}$ is also used as a precipitant in DNA and protein purification as well as for concentrating viruses. PEG is soluble in water, and in many organic solvents, such as ethanol, toluene, chloroform, and acetone. ${ }^{29}$ Paper 2 characterized plasticized polyethylene glycol with the required properties for use as a sacrificial material suitable for syringe-based 3D-printing.

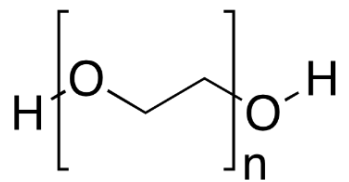

\section{Figure 7 Polyethylene glycol (PEG)}

Addition of plasticizer to PEG decreases the viscosity and thus enables printing of PEGs with higher molecular weight. A plasticizer typically reduces the degree of crystallinity, making the polymer more flexible. $100 \%$ PEG with molecular weight above $2000 \mathrm{~g} / \mathrm{mol}$ (PEG2000) is mechanically stable enough to function as a support, but too rigid to extrude through a syringe tip at room temperature without 
the addition of a plasticizer. The addition of a plasticizer also makes the viscosity of the PEG2000 blend less susceptible to small temperature changes (in the range of $20 \pm 5^{\circ} \mathrm{C}$ ) than PEG with lower molecular weight (e.g. $1000 \mathrm{~g} / \mathrm{mol}$ ). This simplifies handling without risking deformation, where e.g. PEG1000 melts at body temperature when handled. PEG combined with organic carbonates, such as diethyl carbonate (DEC), dimethyl carbonate (DMC), and propylene carbonate (PC), as plasticizer have previously been used as electrolytes in Li polymer batteries ${ }^{31}$ and other ion-conducting applications. ${ }^{28}$ These carbonates were used as plasticizers in paper 2 to make high molecular weight PEG suitable for syringe-based printing. The chemical structures of DEC, DMC, and PC can be seen in Figure 8. As PEG and $\mathrm{PC}$ are both non-toxic and water-soluble they are suitable for biological applications.
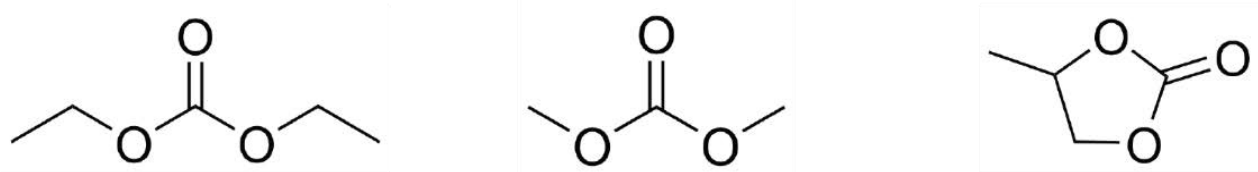

Figure 8 Chemical structures (from left to right) diethyl carbonate (DEC), dimethyl carbonate (DMC), and propylene carbonate (PC) 


\section{Material characterization techniques}

A material suitable for 3D-printing should have the properties described in chapter 5 , e.g. chemical and mechanical stability. The material's flow rate varies if the material's consistency changes with temperature, resulting in uneven printed structures. The viscosity of a material for syringe-based 3D-printing should therefore be stable within $5{ }^{\circ} \mathrm{C}$ of the operating temperature of the printer (usually room temperature) for consistent results. The temperature dependence and consistency of a material can be evaluated and characterized by differential scanning calorimetry and rheometry. Differential scanning calorimetry can measure the temperature at which phase-changes occur. The rheological behavior predicts how easily the material can be extruded during syringe-based 3D printing and how well the material will retain its shape after extrusion. The consistency can be quantified using a rheometer as described in chapter 6.2.

\subsection{Differential scanning calorimetry (DSC)}

Differential scanning calorimetry (DSC) can be used to measure at which temperature a material undergoes a phase transition, i.e. glass transition temperature $\left(\mathrm{T}_{\mathrm{g}}\right)$, melting temperature $\left(\mathrm{T}_{\mathrm{m}}\right)$, and crystallization temperature $\left(\mathrm{T}_{\mathrm{c}}\right) .^{32-}$ ${ }^{34}$ DSC measures the energy transferred as heat by comparing the temperature of the sample with the temperature of a reference at a constant pressure. An electronic feedback controller then tries to adjust the temperature of the reference and the sample in order to maintain the same temperature. This is done while scanning over a range of temperatures in a controlled manner. The temperature change, $\Delta T$, between the sample and the reference is converted into heat, $q$, according to: $q=$ $C_{p} \Delta T$, where $C_{p}$ is the specific heat capacity at a constant pressure. The heat is then plotted versus temperature as in Figure 9. The peaks in Figure 9 are the heat that is either released or absorbed when the material undergoes a chemical or physical process at a constant pressure. 


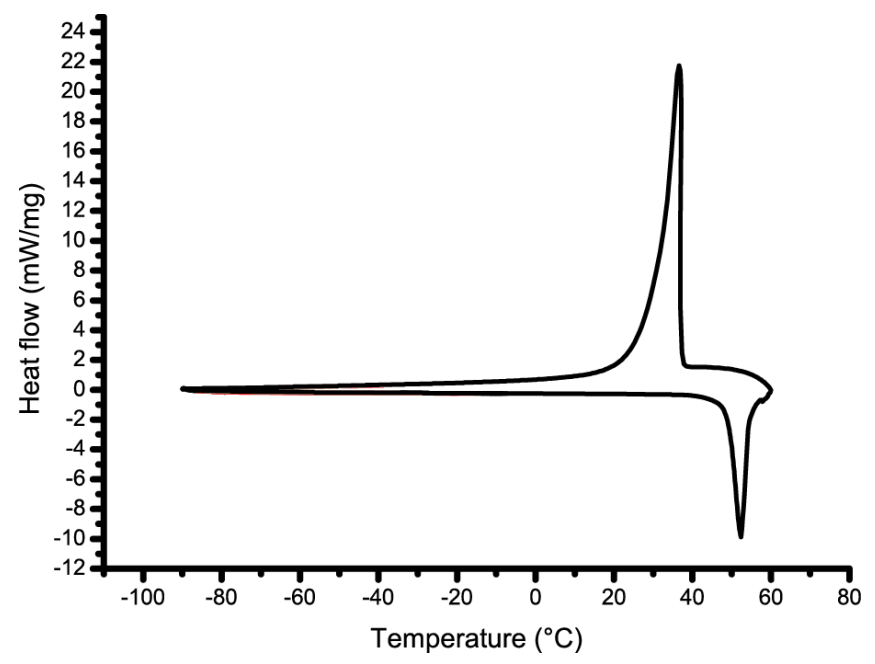

Figure 9 Heat flow (q, $\mathrm{mW} / \mathrm{mg}$ ) versus temperature $\left({ }^{\circ} \mathrm{C}\right)$ measured by differential scanning calorimetry. The peak at $\mathrm{T}=31{ }^{\circ} \mathrm{C}$ indicates crystallization and at $\mathrm{T}=52{ }^{\circ} \mathrm{C}$ indicates melting. The difference in peak size between the forward and reverse sweep are caused by the different temperature scan rates.

\subsection{Rheology}

Rheology is the study of how materials deform when a force is applied. Rheological measurements give information about the mechanical properties of a material, e.g. viscosity and elasticity. For 3D printing, the viscosity, $\eta$, of the material is the most significant parameter for predicting how easily a material can be extruded, including the amount of force required to initiate flow. Viscosity is a liquid's resistance to flow and usually depends on temperature. Liquids are either said to be Newtonian or non-Newtonian. Newtonian liquids have a constant viscosity while the viscosity of non-Newtonian liquids, e.g. ketchup or blood, depends on the shear rate. Shear rate, $\dot{\gamma}\left(\mathrm{s}^{-1}\right)$, is the time-derivative of strain, $\gamma$, defined as, $\dot{\gamma}=\frac{d \gamma}{d t}=\frac{\Delta v}{\Delta z}$, see Figure 10. Strain, $\gamma=\frac{\Delta x}{h}$ (see Figure 11), is the relative deformation, $\Delta \mathrm{x}$, in a specified direction under shear stress, $\tau\left(\mathrm{N} / \mathrm{m}^{2}\right)$. Shear stress is the force per unit area causing the deformation (see Figure 11). 


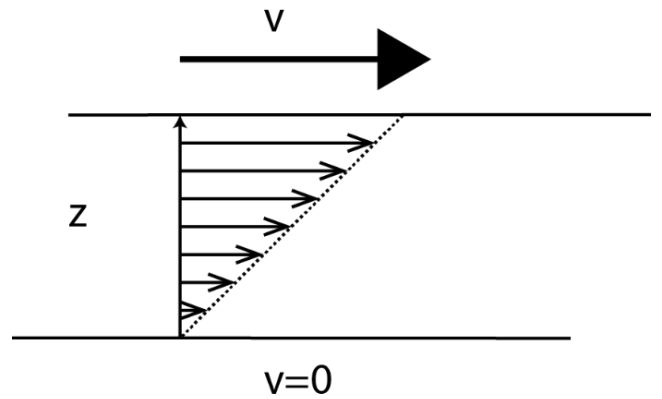

Figure 10 Illustration of parameters used to estimate the shear rate, $\dot{\gamma}=\frac{d \gamma}{d t}=\frac{\Delta v}{\Delta z}$, where the material's velocity, $v$, changes (defined as $\Delta v$ ) from the edge and up to the deformation plane in zdirection, where each step is increased by $\Delta z$.

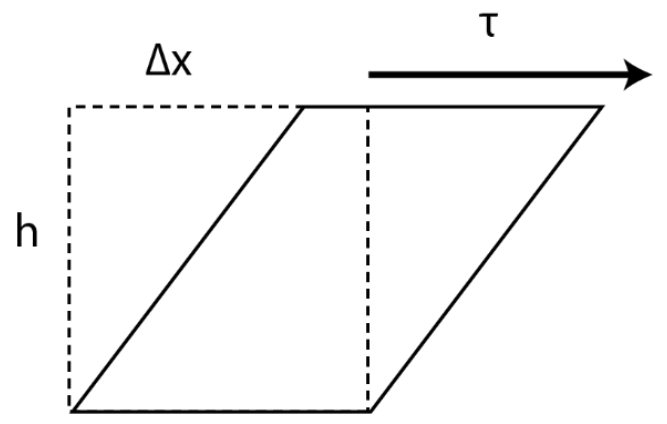

Figure 11 Illustration of strain, $\gamma=\frac{\Delta x}{h}$, which defines the deformation, $\Delta \mathbf{x}$, at a certain shear stress, $\tau\left(\mathbf{N} / \mathbf{m}^{2}\right)$. 
As stated above, most materials are non-Newtonian, meaning that the viscosity changes depending on the shear rate (see Figure 12). If a material's viscosity decreases with increasing shear rate it is said to be shear-thinning. The viscosity changes at a critical shear rate and either decreases for shear-thinning materials or increases for shear-thickening materials and continues to change with increasing shear rate.

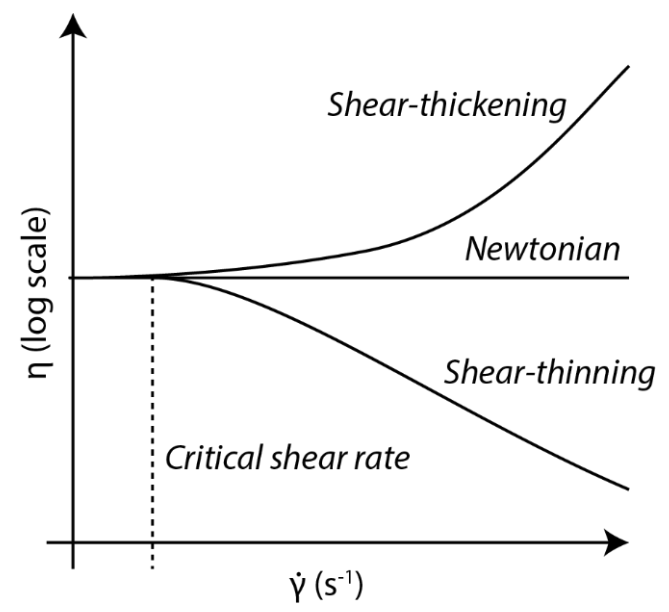

Figure 12 Dynamic viscosity, $\eta$ (Pas), behavior of shear-thickening, Newtonian and shear-thinning material as function of shear rate, $\dot{\gamma}\left(\mathrm{s}^{-1}\right)$.

The possibility to extrude a material can be determined by estimating the viscosity and the critical shear rate for a non-Newtonian material. The shear rates depend on the nozzle size and the material flow rate affecting printing of a non-Newtonian material. Shear-thinning materials flow easily as long as the shear rate is above the critical shear rate and the initial viscosity is low enough. This simplifies extrusion of highly viscous materials through a fine tip since the viscosity of a shear-thinning material will be lower while printing, while returning to the higher viscosity when added to the final structure. Higher viscosity materials require larger forces to start and maintain extrusion than low viscosity materials. Also the force required to maintain the flow for a shear-thinning material is lower than for shear-thickening and Newtonian materials. Shear-thickening and Newtonian materials are more difficult to extrude through smaller tips, since the viscosity is either constant or increasing. These materials require larger forces to maintain the flow through the 
nozzle the higher the shear rates are. It is thus useful to be able to estimate shear rates during printing to evaluate a material's suitability in regards of viscosity.

A material flowing through the nozzle of a printer can be modeled as a liquid flowing through a tube, allowing estimation of shear rates, etc. The flow rate, $Q$ $\left(\mathrm{m}^{3} / \mathrm{s}\right)$, in the tube can be found according to $Q=\frac{\bar{v} \pi r^{3}}{2}$. The average velocity, $\bar{v}$ $(\mathrm{m} / \mathrm{s})$, at which the material is flowing out also named as deposition or extrusion rate and $r(\mathrm{~m})$, is the nozzle radius.

The shear rate experienced by an incompressible Newtonian liquid can then be estimated according to $\dot{\gamma}=\frac{Q}{2 \pi d^{3}}$, based on the flow rate, $Q$, through the tube with a diameter, $d$. The result from this estimation can then be used to determine whether a material is shear-thinning within the expected shear rates for different tip sizes and flow rates.

\subsubsection{Rheometry}

A rheometer measures e.g. viscosity and elasticity of a liquid. This can be done by using a rotating disc rheometer. The rotating disc rheometer that measures the torque and the rotational velocity while rotating the one of the disc in contact with the sample. Then by having known vessel geometries can the viscosity or elasticity be inferred. Three types of geometries are usually employed: cone-and-plate, parallel plate, or concentric cylinder (couette), see Figure 13. The concentric cylinder configuration is used for low viscosity liquids that would otherwise run off the plate configurations. The parallel plate geometry is useful for disc-shaped samples. Notice that the shear rate the sample is exposed to varies as a function of radial position, with maximum shear rate at the perimeter and zero shear rate at the center. The cone-plate geometry has a consistent shear rate from the center and out to the edge of the disc. The geometry that is most appropriate depends on the material's viscosity. Smaller geometries should be used for highly viscous material to reduce the risk of slippage between materials and plate, resulting in a torque underestimation. 

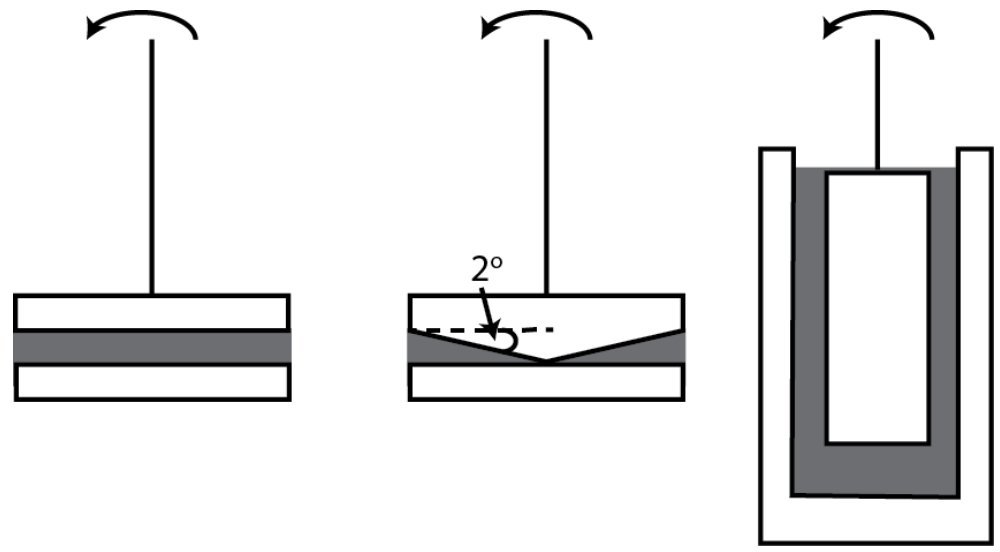

Figure 13 Measurement geometries for rotational rheometers. From left to right: parallel plate, cone-and-plate, and couette. 


\section{Conclusions and future work}

The methods and materials presented in this work can be used to solve some of the issues with miniaturization to facilitate $\mathrm{LoC}$ development. We believe that conducting polymers, and sacrificial materials can be used in combination with 3D-printing to further develop these types of systems. Integration of conductive polymers in microsystem can reduce the effects of water electrolysis due to their electrochemical properties and can easily be adapted to printing. In paper 1, gel electrophoresis is described as an application where conductive polymer electrodes easily can replace metal electrodes without affecting the separation.

Bottom-up manufacture of microsystems using a syringe-based 3D-printer is facilitated by a paste-like sacrificial template material. Combining sacrificial and other materials increase the complexity of printed structures. We show in paper 2 that polyethylene glycol (PEG) combined with propylene carbonate functions as a sacrificial template material suitable for 3D-printing. The consistency of this PEG-based material can be tuned with the amount of plasticizer and can find use in both macroscopic and microscopic applications.

Future work aims to use a syringe-based 3D-printer to produce microsystems for biology and chemistry, where the PEG-based sacrificial material is used to produce molds, support for surrounding structures. All materials used are biocompatible allowing for use in close contact with e.g. cells and biological samples. The next step is to combine the conductive polymer electrodes with the sacrificial template material to print fully functional analytical microsystems. Currently, the printed sacrificial template material and conducting polymers have been combined with soft lithography (molding with polydimethylsiloxane (PDMS)) to produce channels for either cell-culturing or capillary electrophoresis separation to produce the final functional device. The 3D-printer is used to align and assemble channels and electrodes, where the goal is that a customized functional device can be taken straight from the printer, ready for immediate use. 


\section{Summary of included papers}

\subsection{Paper 1}

Conducting polymers for gel electrophoresis

In this study, we evaluated the compatibility of the conductive polymer PEDOT with gel electrophoresis. It was incorporated in a commercially available gel electrophoretic system, the PhastSystem from GE Healthcare. SDS-PAGE was performed with polymer electrodes, where the electrodes showed good compatibility with the gel, buffers and separation protocol. Changing the electrode material from platinum to PEDOT had little impact on the resulting gel image. This study was performed as a first step toward developing smaller, disposable gel electrophoretic systems with incorporated conductive polymer electrodes. We expect that replacing metal electrodes with conducting polymer electrodes, which are cheaper, flexible and electrochemically active, will remove some of the most important obstacles to miniaturization electrophoretic systems.

\subsection{Paper 2}

Sacrificial template materials for syringe-based 3D-printing

In this study, we characterized and evaluated polyethylene glycol (PEG)-based material combined with a plasticizer (diethylene carbonate, dimethyl carbonate or propylene carbonate) for syringe-based 3D-printing. This material is intended for use as a sacrificial material in additive manufacturing to support surrounding materials to facilitate printing of more advanced structures, e.g. overhanging and protruding models, using a syringe-based 3D-printer. PEG-based materials have properties desirable for a sacrificial template such as biocompatibility and watersolubility, and are commercially available in a variety of molecular weight ranges. This type of material can be adjusted to suit certain applications, where e.g. the consistency is tunable with length of polymer chain and amount of plasticizer added.

A suitable blend for syringe-based 3D-printing is a combination of $25-35 \mathrm{wt} \%$ propylene carbonate in PEG2000. This material is easily extruded through a 
syringe, self-supportive with increasing mechanical stability with time after printing and easily dissolved in warm water. Viscosity measurements showed that the viscosity increases with storage time after blending PEG and plasticizer. Differential scanning calorimetry showed that the phase-transition temperatures stay the same regardless of storage time of the material, up to 3 weeks. 


\section{References}

1. Whitesides, G. M. The origins and the future of microfluidics. Nature 442, 368-73 (2006).

2. Lipson, H. \& Kurman, M. Fabricated: The New World of 3D Printing. (2013).

3. Nates, P. B. and S. Gel Electrophoresis - Principles and Basics. (InTech, 2012). doi: $10.5772 / 2205$

4. Berg, J. M., Tymoczko, J. L. \& Stryer, L. Biochemistry. (W.H. Freeman, 2006).

5. Southern, E. Recombinant DNA. Methods in Enzymology 68, (Elsevier, 1979).

6. Shirakawa, H., Louis, E. J., MacDiarmid, A. G., Chiang, C. K. \& Heeger, A. J. Synthesis of electrically conducting organic polymers: halogen derivatives of polyacetylene, $(\mathrm{CH}) \mathrm{x}$. J. Chem. Soc. Chem. Commun. 578 (1977). doi:10.1039/c39770000578

7. Asplund, M. et al. Toxicity evaluation of PEDOT/biomolecular composites intended for neural communication electrodes. Biomed. Mater. 4, 045009 (2009).

8. Isaksson, J. et al. Electronic control of $\mathrm{Ca} 2+$ signalling in neuronal cells using an organic electronic ion pump. Nat. Mater. 6, 673-9 (2007).

9. Wang, X. et al. Evaluation of biocompatibility of polypyrrole in vitro and in vivo. $J$. Biomed. Mater. Res. A 68, 411-22 (2004).

10. Banerjee, S. \& Tyagi, D. A. K. Functional Materials: Preparation, Processing and Applications. (Elsevier, 2012).

11. Groenendaal, L., Jonas, F., Freitag, D., Pielartzik, H. \& Reynolds, J. R. Poly(3,4ethylenedioxythiophene) and Its Derivatives: Past, Present, and Future. Adv. Mater. 12, 481-494 (2000).

12. Kirchmeyer, S. \& Reuter, K. Scientific importance, properties and growing applications of poly(3,4-ethylenedioxythiophene). J. Mater. Chem. 15, 2077 (2005).

13. Erlandsson, P. G. \& Robinson, N. D. Electrolysis-reducing electrodes for electrokinetic devices. Electrophoresis 32, 784-90 (2011).

14. Williamson, A. et al. Controlling Epileptiform Activity with Organic Electronic Ion Pumps. Adv. Mater. 27, 3138-44 (2015).

15. Jager, E. W. H. Conjugated Polymers as Actuators for Medical Devices and Microsystems. 141-161 (2010). 
16. Pei, Q. \& Inganäs, O. Conjugated polymers and the bending cantilever method: Electrical muscles and smart devices. Adv. Mater. 4, 277-278 (1992).

17. Gross, B. C., Erkal, J. L., Lockwood, S. Y., Chen, C. \& Spence, D. M. Evaluation of 3D printing and its potential impact on biotechnology and the chemical sciences. Anal. Chem. 86, 3240-53 (2014).

18. RepRap - RepRapWiki. at $<$ http://reprap.org/wiki/RepRap $>$

19. Jones, R. et al. RepRap - the replicating rapid prototyper. Robotica 29, 177-191 (2011).

20. Malone, E. \& Lipson, H. Fab@Home: the personal desktop fabricator kit. Rapid Prototyp. J. 13, 245-255 (2007).

21. Mannoor, M. S. et al. 3D printed bionic ears. Nano Lett. 13, 2634-9 (2013).

22. Lee, J.-S. et al. 3D printing of composite tissue with complex shape applied to ear regeneration. Biofabrication 6, 024103 (2014).

23. Hockaday, L. A. et al. Rapid 3D printing of anatomically accurate and mechanically heterogeneous aortic valve hydrogel scaffolds. Biofabrication 4, 035005 (2012).

24. Bellan, L. M. et al. Fabrication of an artificial 3-dimensional vascular network using sacrificial sugar structures. Soft Matter 5, 1354 (2009).

25. Müller, M., Becher, J., Schnabelrauch, M. \& Zenobi-Wong, M. Printing thermoresponsive reverse molds for the creation of patterned two-component hydrogels for 3D cell culture. $J$. Vis. Exp. e50632 (2013). doi:10.3791/50632

26. Serra, T., Planell, J. a \& Navarro, M. High-resolution PLA-based composite scaffolds via 3-D printing technology. Acta Biomater. 9, 5521-30 (2013).

27. Billiet, T., Vandenhaute, M., Schelfhout, J., Van Vlierberghe, S. \& Dubruel, P. A review of trends and limitations in hydrogel-rapid prototyping for tissue engineering. Biomaterials 33, 6020-41 (2012).

28. Sun, B., Tehrani, P., Robinson, N. D. \& Brandell, D. Tailoring the conductivity of PEObased electrolytes for temperature-sensitive printed electronics. J. Mater. Sci. 48, 57565767 (2013).

29. Salamone, J. C. Polymeric Materials Encyclopedia, Twelve Volume Set. (CRC Press, 1996).

30. Krsko, P. \& Libera, M. hydrogels Poly ( ethylene glycol ), or PEG, is used extensively in biomedical device. 8, 36-44 (2005). 
31. Do, J. Electrochemical properties of lithium salt-poly(ethylene oxide)-ethylene carbonate polymer electrolyte and discharge characteristics of Li/MnO2. Solid State Ionics 89, 291298 (1996).

32. Gill, P., Moghadam, T. T. \& Ranjbar, B. Differential scanning calorimetry techniques: applications in biology and nanoscience. J. Biomol. Tech. 21, 167-93 (2010).

33. Handbook of Thermal Analysis and Calorimetry: volume 1 Principles and Practice. (Elsevier, 1998).

34. Atkins, P. \& de Paulo, J. Atkin's physical chemistry. (Oxford University press). 


\section{Papers}

The articles associated with this thesis have been removed for copyright reasons. For more details about these see:

http://urn.kb.se/resolve?urn=urn:nbn:se:liu:diva-121252 